\title{
Do the New Hydrophilic Surface Have Any Influence on Early Success Rate and Implant Stability during Osseointegration Period? Four-Month Preliminary Results from a Split-Mouth, Randomized Controlled Trial
}

\author{
Marco Tallarico ${ }^{1}$ Nicola Baldini ${ }^{2}$ Matteo Martinolli ${ }^{3}$ \\ Silvio Mario Meloni ${ }^{6}$ \\ 1 Implantology and Prosthetic Aspects, Master of Science in \\ Dentistry Program, Aldent University, Tirana, Albania \\ 2 Department of Periodontics and Implantology, University of Siena, \\ Siena, Italy \\ ${ }^{3}$ Private Practice, University of Padova, Porto Viro, Italy \\ ${ }^{4}$ Department of Oral and Maxillofacial Surgery, Insan Apsun Dental \\ Clinic, South Korea \\ ${ }^{5}$ Department BIOMORF, School of Dentistry, University of Messina, \\ Messina, Italy \\ ${ }^{6}$ Department of Surgical, Microsurgical and Medical Science, \\ University of Sassari, Sassari, Italy
}

Eur J Dent 2019;13:95-101

\section{Erta Xhanari ${ }^{1}$ Yong-Jin Kim ${ }^{4}$ Gabriele Cervino ${ }^{5}$}

\begin{abstract}
Address for correspondence Marco Tallarico, Adjunct professor, Implantology and Prosthetic Aspects, Master of Science in Dentistry Program, Aldent University, Rr.e Dibrës, Nr. 235, Tirana, Albania (e-mail: me@studiomarcotallarico.it).
\end{abstract}

Abstract

Keywords

- dental implant

- early loading

- implant stability quotient

- implant surface

- osseointegration
Objective The objective of this study is to compare the implant stability of Hiossen ET III implants with its new hydrophilic (NH) surface and Hiossen ET III implants with the sandblasted and acid-etched (SA) surface.

Materials and Methods Patients required at least two teeth to be rehabilitated with a fixed, implant-supported restoration, consecutively enrolled. Patients randomly received SA surface implants (SA group) or SA implants with a newly developed bioabsorbable apatite nanocoating ( $\mathrm{NH}$ group). Outcome measures were implant and prosthetic survival rate, complications, insertion torque, and implant stability quotient (ISQ) measured at implant placement and every week up to 8 weeks after implant placement. Comparison between groups was made by unpaired $t$-test, while the comparison between each follow-up will be made by paired $t$-tests to detect any change during the follow-up. Complications and failures were compared using Fisher's exact test.

Results A total of 14 patients were treated with 28 implants (14 SA and $14 \mathrm{NH}$ ). No implant and prosthesis failed 4 months after implant placement. No complications were experienced. At the 2 nd week after implants placement, two implants in the SA group showed discontinuous measurements versus none in the $\mathrm{NH}$ group $(p=0.4815)$. Implants unscrewed during ISQ measurements and were rescrewed. Data recording stopped for 6 weeks. Both implants osseointegrated without any further complication. The NH implants did not show physiological ISQ decrease between 2nd and 4th week after implant placement, showing a more even pattern of ISQ values compared with SA implants (77.1 \pm 4.6 vs. $72.9 \pm 11.5$; difference: $4.2 \pm 12.1 ; p=0.258)$. High ISQ values were found in both groups at each time point.

Conclusions NH implants are a viable alternative to SA surface, as they seem to avoid the ISQ drop during the remodeling phase.
DOI https://doi.org/

$10.1055 / \mathrm{s}-0039-1688737$

ISSN 1305-7456.
License terms

() (1) $\Theta \circledast$ 


\section{Introduction}

Nowadays, the use of dental implants has become an accepted treatment modality in clinical dentistry in both fixed and removable solutions. ${ }^{1,2,3,4,5}$ Over the years, implant designs and surgical techniques have undergone significant improvements, resulting in current survival rates surpassing $95 \%$ after 5 years of follow-up. ${ }^{6}$

Today, clinical research is focusing on shorter and less invasive procedures. Different implant designs, ${ }^{7.8}$ as well as, placement and loading protocols are currently used to shorten treatment times and decrease the amount of surgical interventions, enabling clinicians to choose between a one(nonsubmerged) and a two-stage (submerged) approach. Against different loading protocol, it has been showed that the submerged technique is not a prerequisite for osseointegration, ${ }^{9}$ even if one-stage implant placement might be at a slightly higher risk of early failures. Primary implant stability is still considered to be a prerequisite for the longterm success of an implant-supported prosthesis. ${ }^{10}$ Primary stability depends mainly on the macro- and micro-design of the implant including the functional length, besides surgical technique and properties of local bone. ${ }^{11,12}$

The more their applications increase, the greater the clinical interest becomes in the implants integrating quickly with the bone to be functional. In the last decade, there was an ongoing effort to improve the interface between bone and implant to speed up the process of osseointegration and improve its quality. ${ }^{13}$ These efforts have been concentrating in improving this interface chemically (by incorporating inorganic phases on or into the titanium oxide layer) or physically (by increasing the level of roughness). ${ }^{14}$

Different techniques have been utilized to alter the surface topography of dental implants. These techniques are usually applying either additive or subtractive concepts. Long-term studies showed that additive surfaces have a higher incidence of complications which was attributed to the delamination of the thick HA layer and to the uncontrolled rate of dissolution of deposited phases. ${ }^{15}$ Consequently, subtractive surfaces have become more popular by clinicians.

Although shorter healing period was presented in many experimental and clinical studies using sandblasted and acidetched (SLA) surfaces, ${ }^{16,17}$ modification of this surface seems to presents a stronger bone response than its predecessor. ${ }^{18,19}$

The purpose of this split-mouth randomized controlled trial was to compare early implant failure and implant stability of one-stage Hiossen ET III implants with its new hydrophilic (NH) surface, compared with Hiossen ET III implants with the well-known SA surface. The null hypothesis was that there is no difference between groups. The null hypothesis was tested against the alternative hypothesis of differences between them. The following trial was reported according to the STROBE statement.

\section{Materials and Methods}

This study was designed as a split-mouth, randomized controlled trial of parallel groups with two arms, conducted at one center, between November 2017 and May 2018. The protocol was registered in the clinicaltrial.gov (NCT03649100). The 2013 Helsinki declaration was adhered too. The study was performed after approval was received from the Institutional Review Board of the Aldent University, Tirana, Albania (3/2018). Surgical and prosthetic procedures were performed by one expert clinician.

Any healthy patients, aged 18 years or older, required at least two implants to be rehabilitated with a fixed implant-supported restoration, with a full mouth bleeding and full mouth plaque index $\leq 25 \%$, with a sufficient bone to allow placement of at least $11.5 \mathrm{~mm}$-long implants, and bone width of at least 6 to $8 \mathrm{~mm}$ for the placement of a regular platform Hiossen ET III implant (Deutsche Osstem GmbH, Eschborn, Germany) were included in this study.

The exclusion criteria were as follows: positive medical findings (such as stroke, recent cardiac infarction, severe bleeding disorder, uncontrolled diabetes, or cancer), psychiatric therapy, pregnancy or nursing, smoking $>10$ cigarettes per day, insertion torque $<35 \mathrm{Ncm}$, untreated periodontitis, acute and chronic infections of the adjacent tissues or natural dentition, previous radiotherapy of the oral and maxillofacial region within the past 5 years, postextractive implants (at least 3 months after tooth extraction), absence of teeth in the opposing jaw, severe clenching or bruxism, severe maxillomandibular skeletal discrepancy, and poor oral hygiene.

Patients were informed about the clinical procedures, the materials to be used, the benefits, potential risks and complications, as well as any follow-up evaluations required for the clinical study. Patients had to sign the informed consent before including in the study.

A single dose of antibiotic ( $2 \mathrm{~g}$ of amoxicillin and clavulanic acid or clindamycin $600 \mathrm{mg}$ if patients were allergic to penicillin) was administered prophylactically 1 hour before surgery. Patients rinsed with $0.2 \%$ chlorhexidine for 1 minute. Local anesthesia will be induced using a $4 \%$ articaine solution with epinephrine 1:100 000 (Ubistesin; 3M Italia, Milan, Italy). Implants were placed in the planned anatomic sites using a flapless or a mini-flap approach ( - Figs. 1 and 2). Bone density was assessed, according to the Lekholm and Zarb classification, during the drilling phase, based on the clinician's experience and judgment. Implant site was prepared simultaneously, according to the drilling protocol recommended by the manufacturer. SA

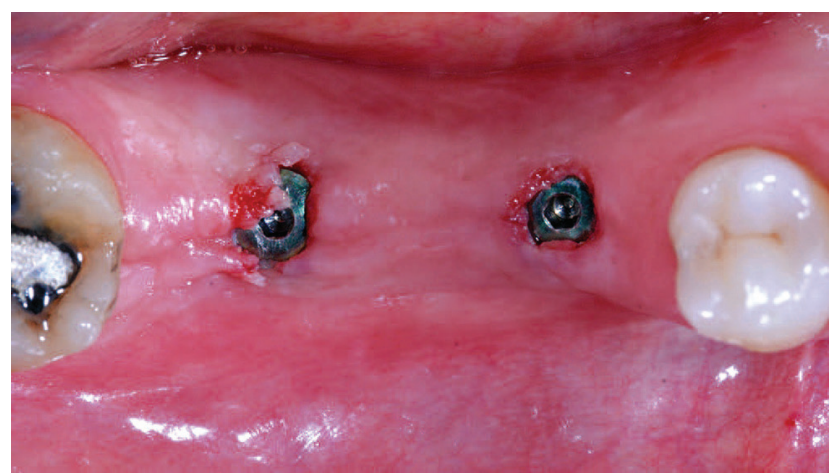

Fig. 1 Computer-assisted template-based implant placement (Osstem OneGuide Kit, Osstem, Seoul, South Korea): occlusal view. 


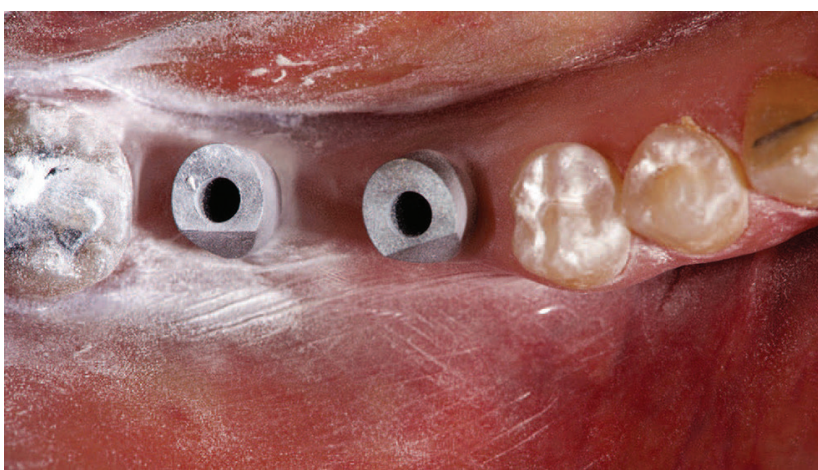

Fig. 2 Digital impression (3M true definition scanner, 3M Italia): occlusal view.

surface implants (SA group) or SA surface implants with a newly developed bioabsorbable apatite nanocoating ( $\mathrm{NH}$ group) were randomized after implant site preparation, immediately before implant placement. Implants used in every group were identical except for the surface treatment. After implant placement, the insertion torque values of the implants were measured and recorded during surgery using a surgical unit (iChiroPro, Bien Air, Italy). Then, a smart peg (Type 47 cod. 100478, Osstell, Gothenburg, Sweden) was connected to the implants, and the implant stability quotient (ISQ) was measured and recorded using the Osstell Mentor device (Osstell), at implant placement, and every week up to 8 weeks after implant placement.

Implants were placed according to a one-stage protocol and measured every week up to 8 weeks. Then implants were measured again 12 weeks after implant placement. In case of ISQ value $<55$ or in case of implant mobility, healing abutment was replaced with a cover screw and the implant was left to heal submerged for at least 6 weeks.

Postsurgical analgesic treatment was performed with ibuprofen $600 \mathrm{mg}$, which was administered twice a day for 2 days after the surgery, and later on, if required. Periapical radiographs were taken with a customized holder at implant placement, at the definitive prosthesis delivery, and then yearly. Two to three months after implants placement patients receive single screw-retained restorations (-Figs. 3-9).

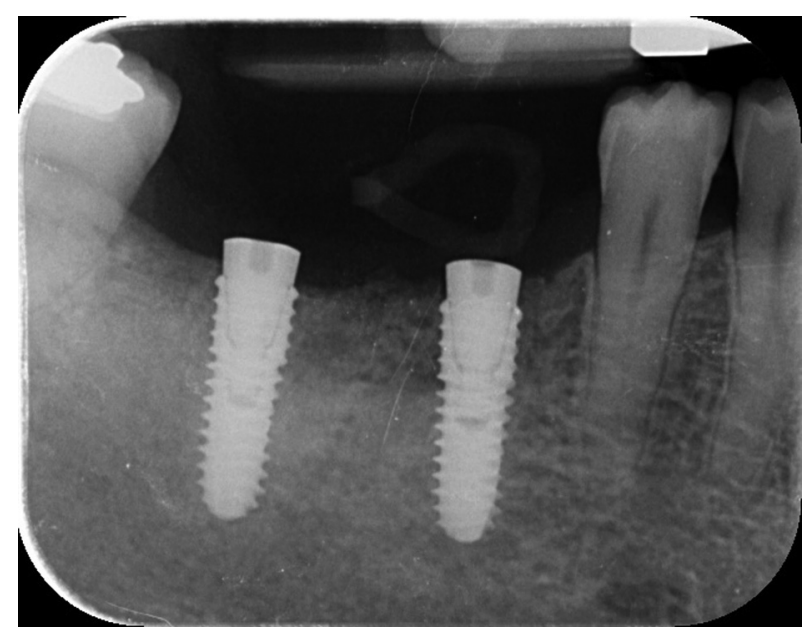

Fig. 3 Periapical radiograph at implant placement.

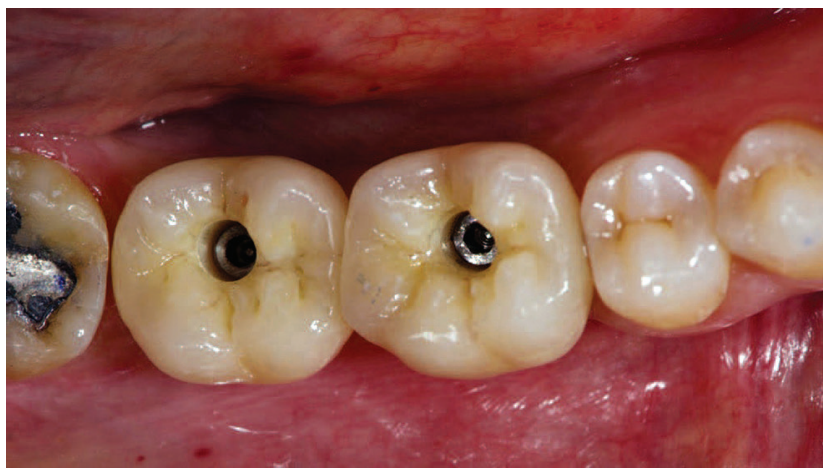

Fig. 4 Definitive, single, screw-retained crowns: occlusal view. The distal implant was left to heal submerged.

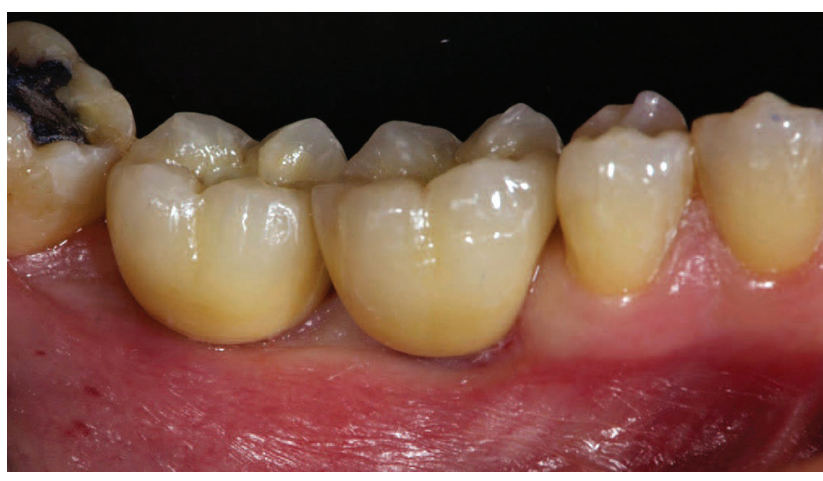

Fig. 5 Definitive, single, screw-retained crowns: lateral view. The distal implant was left to heal submerged.

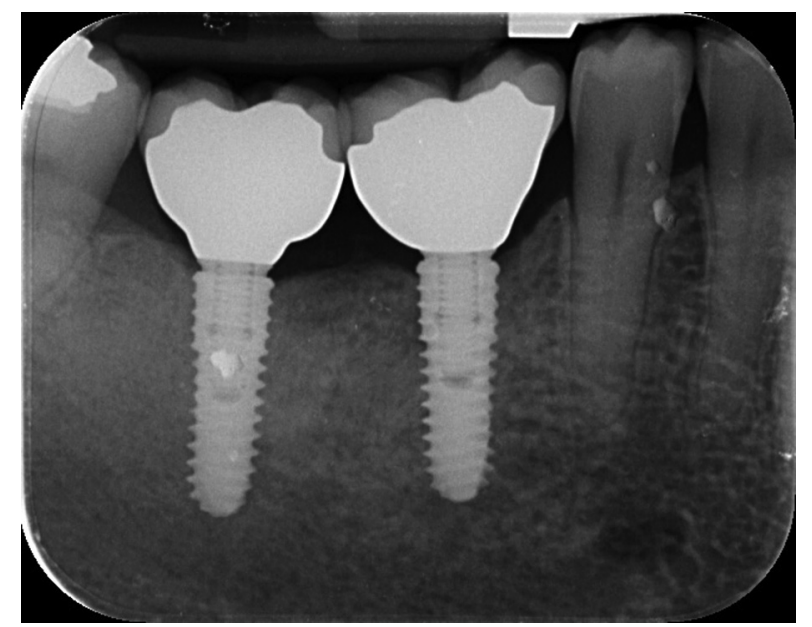

Fig. 6 Periapical radiograph at definitive crowns delivery. The distal implant was left to heal submerged.

The outcome measures were implant and prosthetic survival rates, any biological or mechanical complications at implants that occurred during the entire observation period.

Success rates of the implants and prostheses were evaluated by an independent assessor (EX). An implant was considered a failure if it presented mobility, assessed after the osseointegration period by tapping or rocking the implant head with the metallic handles of two instruments, progressive marginal bone loss or infection, or any mechanical 


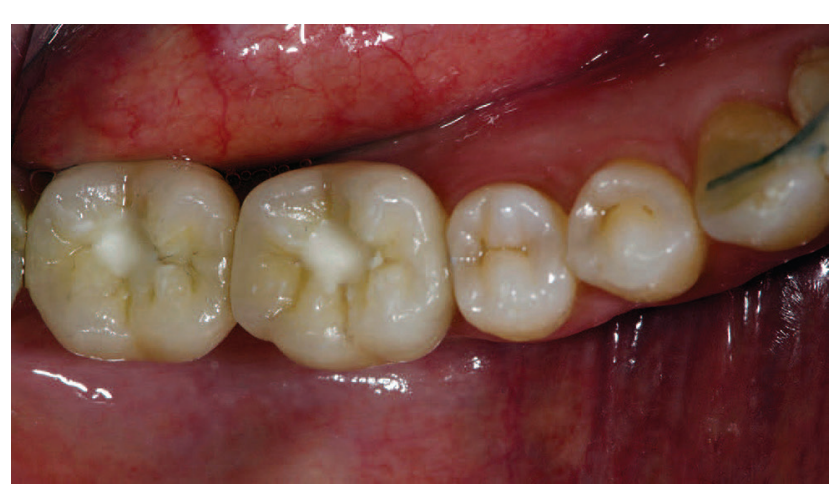

Fig. 7 Definitive restorations at 6-month of follow-up: occlusal view.

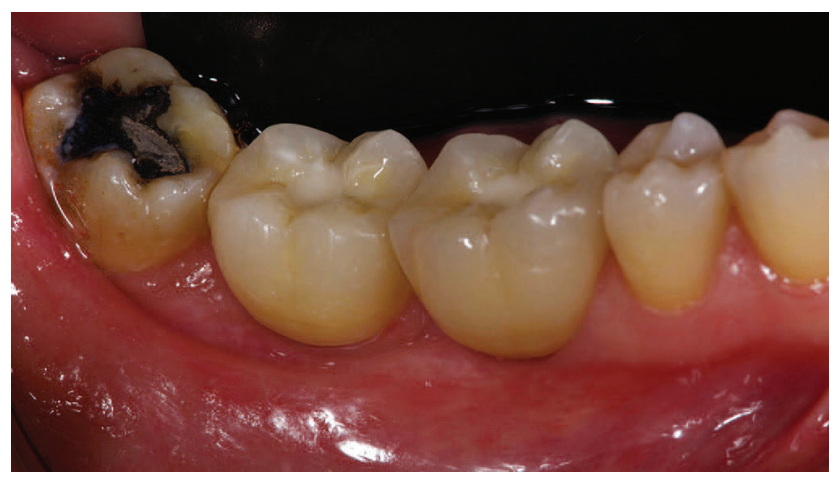

Fig. 8 Definitive restorations at 6-month of follow-up: lateral view.

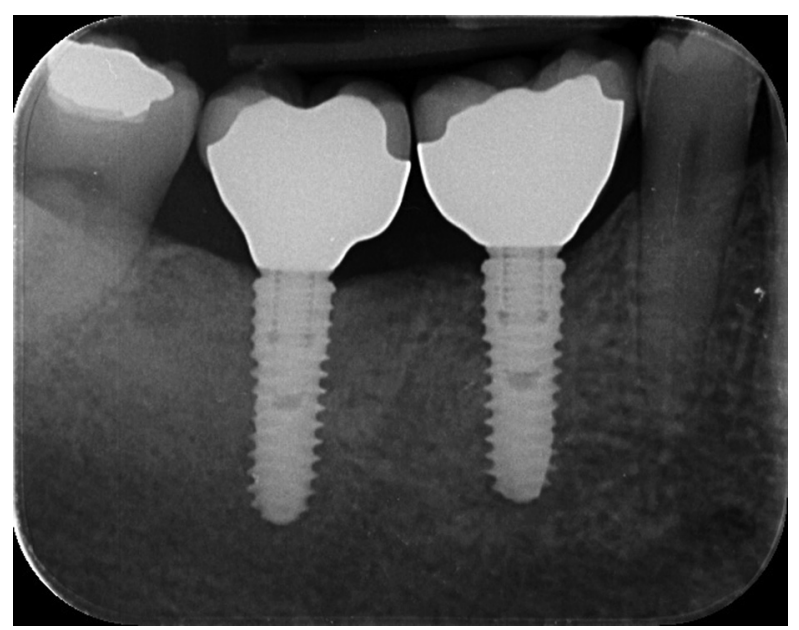

Fig. 9 Periapical radiograph at 6-month of follow-up.

complications rendering the implant unusable, although still mechanically stable in the bone. A prosthesis was considered a failure if it needed to be replaced with another prosthesis.

Biological (pain, swelling, suppuration, etc.) and/or mechanical (screw loosening, fracture of the framework, the veneering material, etc.) complications occurred during the follow-up period. Complications were evaluated and treated by the same surgeon (MT).

Insertion torque was recorded at implant placement by the same surgeon (MT) using the iChiropro surgical unit (Bien-Air, Bienne, Switzerland).
ISQ values were recorded each week up to 8 weeks, and then, after 12 weeks, using resonance frequency analysis (Osstell Mentor device, Osstell, Gothenburg, Sweden), according to a previously published study. ${ }^{2} \mathrm{~A}$ blind outcome assessor collected the data (EX).

A pregenerated random list, consisting of a randomized sequence of consecutive numbers matching the two different procedures within group A or group B, was created using random number generator pro 1.91 for Windows (Segobit Software; www.segobit.com). Opaque envelopes containing the randomization codes were sequentially numbered and sealed. According to a pregenerated list, an independent consultant, not previously involved in the trial, prepared all the envelopes and then opened immediately after implant sites preparation. Site one was defined the site with the lower sextant number and the most mesial. Patient data were collected in an Excel spreadsheet (Microsoft) that reflected the parameters in the patient records. The data were exported into SPSS software for Mac OS X (version 22.0; SPSS, Chicago, Illinois, United States), for the statistical analysis. Descriptive analysis was performed for numeric parameters using means and standard deviations (95\% confidence interval). Comparison between groups was made by unpaired $t$-test, while the comparison between each follow-up will be made by paired $t$-tests to detect any change during the follow-up. Complications and failures were compared using the Fisher's exact test. All statistical comparisons were two-tailed and conducted at the 0.05 level of significance. The patient was used as the statistical unit of analysis.

\section{Results}

A total of 14 patients ( 13 females and 1 male, with a men age of $58.3 \pm 11.9$ ) were screened as they were consecutively enrolled for the trial. All patients were originally treated according to the allocated interventions and no patient dropped out. A total of 28 implants ( 14 with SA surface and 14 with SA surface with the newly developed bioabsorbable apatite nanocoating) were placed. Four patients were rehabilitated in the mandible and 10 in the maxilla. Four-month after definitive prosthesis delivery, no implant and no prosthesis failed. Two weeks after placement, two Hiossen ET III SA implants showed a small mobility with ISQ values lower than 55 (49 and 51, respectively) while no complications were reported in the NH group. Nevertheless, no statistically significant difference was reached $(p=0.4815)$. In both the implants, the healing abutments were replaced with a cover screw and the implants were left to heal submerged for 6 weeks.

The overall insertion torque ranged between 35.0 and 45.0 $\mathrm{Ncm}$ (mean of $41.5 \pm 3.3$ [39.7-43.5] Ncm in the SA group and $41.4 \pm 3.2[40.3-43.9] \mathrm{Ncm}$ in the $\mathrm{NH}$ group). The difference was not statistically significant $(p=0.936)$.

The ISQ values between groups and within time were reported in - Table 1. Although there is no statistically significant difference between groups, $\mathrm{NH}$ implants did not show physiological ISQ decrease between 2nd and 4th week after implant placement, showing a more even pattern of ISQ values (-Fig. 10). At the 2nd week, Hiossen ET III implants with its $\mathrm{NH}$ group showed a mean ISQ value of $77.1 \pm 4.6$ 
Table 1 Implant stability quotient value between groups

\begin{tabular}{|l|l|l|l|}
\hline Weeks & SA $(n=14)$ & NH $(n=14)$ & $p-$ value \\
\hline 0 & $77.9 \pm 5.9(76.2-82.8)$ & $76.7 \pm 5.6(71.6-78.4)$ & 0.611 \\
\hline 1 & $77.2 \pm 5.6(76.4-82.6)$ & $77.4 \pm 5.3(73.3-79.2)$ & 0.941 \\
\hline 2 & $72.9 \pm 11.5^{a}(71.5-84.5)$ & $77.1 \pm 4.6(73.4-78.6)$ & 0.258 \\
\hline 3 & $76.9 \pm 4.6^{a}(72.9-78.1)$ & $77.3 \pm 4.7(74.8-80.2)$ & 0.863 \\
\hline 4 & $78.4 \pm 3.6^{\text {a }}(76.0-80.0)$ & $77.5 \pm 4.3(75.1-79.9)$ & 0.582 \\
\hline 5 & $78.6 \pm 3.1^{\text {a }}(76.3-79.8 .8)$ & $77.8 \pm 4.1(75.7-80.3)$ & 0.604 \\
\hline 6 & $78.7 \pm 3.9^{a}(76.0-80.5)$ & $78.0 \pm 4.2(75.6-80.4)$ & 0.694 \\
\hline 8 & $78.1 \pm 5.1(75.9-81.2)$ & $79.2 \pm 3.9(77.8-82.2)$ & 0.576 \\
\hline
\end{tabular}

Abbreviations: $\mathrm{NH}$, new hydrophilic; SA, sandblasted and acid-etched.

aTwo implants were left to heal submerged and were not measured $(n=12)$.

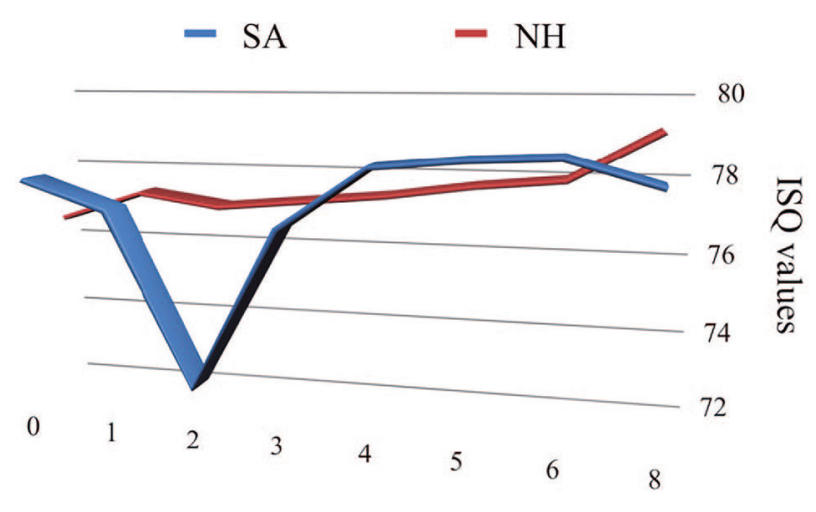

Weeks

Fig. 10 Development of the implant stability quotient values in the sandblasted and acid-etched and new hydrophilic surface group during the 8 weeks of study period. ISQ, implant stability quotient; $\mathrm{NH}$, new hydrophilic; SA, sandblasted and acid-etched.

(73.4-78.6) compared with $72.9 \pm 11.5$ (71.5-84.5) of the Hiossen ET III implants with the well-known SA surface. The difference was not statistically significant $(4.2 \pm 12.1$ [-6.3-7.3]; $p=0.258$ ). Compared with the baseline (implant placement), at the last follow-up examination the $\mathrm{NH}$ implants showed a little improvement in the ISQ values (76.7 \pm 6.0 [71.6-78.4] compared with $79.2 \pm 3.9$ [77.8-82.2]; difference $2.5 \pm 4.3$ [0.1-4.9]; $p=0.246$ ) compared with the SA implants (78.0 \pm 5.9 [76.2-82.8] compared with $78.1 \pm 5.1$ [75.9-81.6]; difference $0.2 \pm 2.3$ [-1.1-1.6]; $p=0.941$ ). The differences were not statistically significant.

\section{Discussion}

This split-mouth randomized controlled trial was designed to evaluate if the new SA surface with a newly developed bioabsorbable apatite nanocoating has any influence on early success rate and implant stability during osseointegration period. The results of the present study have not shown any statistically significant difference in ISQ measurements even though $\mathrm{NH}$ implants did not show physiological ISQ decrease between 2 nd and 4 th week after implant placement, showing a more even pattern of ISQ values.
Primary stability and absence of micromovements are two of the main prerequisites for obtaining a stable osseointegration and the achievement of long-term high-success rates for dental implants. ${ }^{20,21}$ In fact, if primary stability is absent during the early healing period, implant mobility can occur, and this could lead to a soft-tissue interface promoting its failure. ${ }^{22,23}$ In the last decades to reduce the risk of soft-tissue encapsulation, it has been recommended that implants be kept load-free during a healing period of 3 to 4 months in mandibles and 6 to 8 months in maxillae. ${ }^{24}$

Nowadays, the more implants are used in clinical routine, the greater the clinical interest becomes in the implants integrating quickly with the bone to be functional. An ongoing effort to improve the interface between bone and implant surface to speed up the process of osseointegration has been proposed by researcher and dental implant companies.

Commonly, implant surface roughness is divided, depending on the dimension of the measured surface features, into macro-, micro-, and nanoroughness. Typically, these different roughness features are related to distinct effects during wound healing and osseointegration..$^{25}$ Titanium nanostructures have been created by different approaches such as oxidative nanopatterning by acid etching in mixtures of sulfuric acid and hydrogen peroxide, by exposing titanium samples to flowing synthetic air, electrochemically by anodic oxidation, by processing samples after acid etching under protective gas and storing them in saline, by plasma etching, or by physical vapor deposition techniques. ${ }^{26}$

It is well known that roughness increases implant osseointegration, and several implant types are sandblasted and/ or acid-etched to increase their surface texture. ${ }^{22}$ Nevertheless, surfaces coated with hydroxyapatite (HA) were reported to have a higher incidence of complications, ${ }^{15}$ even if evidence for the influence of the implant surface characteristics as a risk indicator for peri-implantitis is very limited. ${ }^{27}$

Vice versa, the nanometer roughness plays an important role in the adsorption of proteins, adhesion of osteoblastic cells, and thus the rate of osseointegration..$^{28}$ In fact, the deposition through dip coating of nanocomposite $\left(\mathrm{HA}-\mathrm{ZrO}_{2}-\mathrm{Al}_{2} \mathrm{O}_{3}\right)$ on titanium substrate showed the highest adhesion strength compared with the HA coatings. ${ }^{29}$ Furthermore, Schwarz et al showed that angiogenesis was enhanced on hydrophilic 
surfaces during early stages of osseointegration. ${ }^{30,31}$ Actually, fast vascularization seems beneficial for bone formation because osteogenic cells have been observed to arise from pericytes adjacent to small blood vessels..$^{21,32}$

In the present study, implants with the hydrophilic surface seem to avoid the ISQ drop during the remodeling phase allowing accordingly benefits in immediate loading, poor bone quality, postextractive, smoking, and immunosuppression disease. Looking for a deeper understanding of the different bioresponses to SLActive versus SLA, a very recent study found thinner carbon contamination films on SLActive $(0.8 \mathrm{~nm})$ compared with SLA (1.6 nm) suggesting an impact of the different contamination films on the blood response, leading to accelerated osseointegration. ${ }^{33}$

In a review of the available human studies, Wennerberg et al have found little clinical evidence so far to clearly state a preference for SLActive over SLA implant. ${ }^{34}$ In a splitmouth study, SLActive implants were compared with SLA implants with early loading protocols in irradiated patients. One-hundred two implants were placed in 20 patients in both jaws. At 1-year follow-up, there was a high survival rate ( $100 \%$ for SLActive vs. $96 \%$ for SLA implants) and low crestal bone loss $<0.4 \mathrm{~mm}$ in both groups with no significant difference. ${ }^{35}$

\section{Conclusions}

High ISQ values were found in both groups at each time point. NH implants are a viable alternative to SA surface, as they seem to avoid the ISQ drop during the remodeling phase. It can be beneficial in immediate loading, poor bone quality, postextractive, smoking, and immunosuppression. Further trials with larger sample size and longer follow-up are needed to confirm these preliminary results.

\section{Financial Support and Sponsorship}

Nil.

\section{Conflicts of Interest}

DEUTSCHE OSSTEM GmbH donated the implants. However, data belonged to the authors and by no means did the company interfere with the conduct of the trial or the publication of its results.

\section{References}

1 Cicciù M, Cervino G, Milone D, Risitano G. FEM investigation of the stress distribution over mandibular bone due to screwed overdenture positioned on dental implants. Materials (Basel) 2018;11(9):E1512

2 Cicciù M, Cervino G, Bramanti E, et al. FEM analysis of mandibular prosthetic overdenture supported by dental implants: evaluation of different retention methods. Comput Math Methods Med 2015;2015:943839

3 Bramanti E, Cervino G, Lauritano F, et al. FEM and Von Mises analysis on prosthetic crowns structural elements: evaluation of different applied materials. Sci World J, 2017;1-7. Article ID 1029574. Available at https://doi.org/10.1155/2017/1029574. Accessed on April 20, 2019

4 Cicciu M, Bramanti E, Matacena G, Guglielmino E, Risitano G. FEM evaluation of cemented-retained versus screw-retained dental implant single-tooth crown prosthesis. Int J Clin Exp Med 2014;7(4):817-825

5 Cicciù M, Risitano G, Maiorana C, Franceschini G. Parametric analysis of the strength in the "Toronto" osseous-prosthesis system. Minerva Stomatol 2009;58(1-2):9-23

6 Yi YS, Emanuel KM, Chuang SK. Short $(5.0 \times 5.0 \mathrm{~mm})$ implant placements and restoration with integrated abutment crowns. Implant Dent 2011;20(2):125-130

7 Lauritano F, Runci M, Cervino G, Fiorillo L, Bramanti E, Cicciù M. Three-dimensional evaluation of different prosthesis retention systems using finite element analysis and the Von Mises stress test. Minerva Stomatol 2016;65(6):353-367

8 Cervino G, Romeo U, Lauritano F, et al. Fem and von mises analysis of OSSTEM ${ }^{\circledR}$ dental implant structural components: evaluation of different direction dynamic loads. Open Dent J 2018;12:219-229

9 Tallarico M, Vaccarella A, Marzi GC. Clinical and radiological outcomes of 1- versus 2-stage implant placement: 1-year results of a randomised clinical trial. Eur J Oral Implantology 2011;4(1):13-20

10 Le Guéhennec L, Soueidan A, Layrolle P, Amouriq Y. Surface treatments of titanium dental implants for rapid osseointegration. Dent Mater 2007;23(7):844-854

11 Glauser R, Sennerby L, Meredith N, et al. Resonance frequency analysis of implants subjected to immediate or early functional occlusal loading. Successful vs. failing implants. Clin Oral Implants Res 2004;15(4):428-434

12 Toyoshima T, Wagner W, Klein MO, Stender E, Wieland M, Al-Nawas B. Primary stability of a hybrid self-tapping implant compared to a cylindrical non-self-tapping implant with respect to drilling protocols in an ex vivo model. Clin Implant Dent Relat Res 2011;13(1):71-78

13 Renvert S, Polyzois I, Claffey N. How do implant surface characteristics influence peri-implant disease? J Clin Periodontol 2011;38(Suppl 11):214-222

14 Dohan Ehrenfest DM, Coelho PG, Kang BS, Sul YT, Albrektsson T. Classification of osseointegrated implant surfaces: materials, chemistry and topography. Trends Biotechnol 2010; 28(4):198-206

15 Piattelli A, Cosci F, Scarano A, Trisi P. Localized chronic suppurative bone infection as a sequel of peri-implantitis in a hydroxyapatite-coated dental implant. Biomaterials 1995;16(12):917-920

16 Payne AG, Tawse-Smith A, Duncan WD, Kumara R. Conventional and early loading of unsplinted ITI implants supporting mandibular overdentures. Clin Oral Implants Res 2002;13(6):603-609

17 Cochran DL, Schenk RK, Lussi A, Higginbottom FL, Buser D. Bone response to unloaded and loaded titanium implants with a sandblasted and acid-etched surface: a histometric study in the canine mandible. J Biomed Mater Res 1998;40(1):1-11

18 Khandelwal N, Oates TW, Vargas A, Alexander PP, Schoolfield JD, Alex McMahan C. Conventional SLA and chemically modified SLA implants in patients with poorly controlled type 2 diabetes mellitus--a randomized controlled trial. Clin Oral Implants Res 2013;24(1):13-19

19 Lee JT, Cho SA. Biomechanical evaluation of laser-etched Ti implant surfaces vs. chemically modified SLA Ti implant surfaces: removal torque and resonance frequency analysis in rabbit tibias. J Mech Behav Biomed Mater 2016;61:299-307

20 Brånemark PI, Hansson BO, Adell R, et al. Osseointegrated implants in the treatment of the edentulous jaw. Experience from a 10-year period. Scand J Plast Reconstr Surg Suppl 1977; $16: 1-132$

21 Makary C, Rebaudi A, Sammartino G, Naaman N. Implant primary stability determined by resonance frequency analysis: correlation with insertion torque, histologic bone 
volume, and torsional stability at 6 weeks. Implant Dent 2012;21(6):474-480

22 Szmukler-Moncler S, Salama H, Reingewirtz Y, Dubruille JH. Timing of loading and effect of micromotion on bone-dental implant interface: review of experimental literature. J Biomed Mater Res 1998;43(2):192-203

23 Lioubavina-Hack N, Lang NP, Karring T. Significance of primary stability for osseointegration of dental implants. Clin Oral Implants Res 2006;17(3):244-250

24 Brånemark PI, Adell R, Breine U, Hansson BO, Lindström J, Ohlsson A. Intra-osseous anchorage of dental prostheses. I. Experimental studies. Scand J Plast Reconstr Surg 1969; 3(2):81-100

25 Junker R, Dimakis A, Thoneick M, Jansen JA. Effects of implant surface coatings and composition on bone integration: a systematic review. Clin Oral Implants Res 2009;20(Suppl 4): 185-206

26 Rupp F, Liang L, Geis-Gerstorfer J, Scheideler L, Hüttig F. Surface characteristics of dental implants: a review. Dent Mater 2018;34(1):40-57

27 Heitz-Mayfield LJ. Peri-implant diseases: diagnosis and risk indicators. J Clin Periodontol 2008;35(8 Suppl :292-304

28 Brett PM, Harle J, Salih V, et al. Roughness response genes in osteoblasts. Bone 2004;35(1):124-133
29 Braceras I, Alava JI, Oñate JI, et al. Improved osseointegration in ion implantation-treated dental implants. Surf Coat Tech 2002;158-159:28-32

30 Schwarz F, Herten M, Sager M, Wieland M, Dard M, Becker J. Histological and immunohistochemical analysis of initial and early osseous integration at chemically modified and conventional SLA titanium implants: preliminary results of a pilot study in dogs. Clin Oral Implants Res 2007;18(4):481-488

31 Long MW, Robinson JA, Ashcraft EA, Mann KG. Regulation of human bone marrow-derived osteoprogenitor cells by osteogenic growth factors. J Clin Invest 1995;95(2):881-887

32 Reilly TM, Seldes R, Luchetti W, Brighton CT. Similarities in the phenotypic expression of pericytes and bone cells. Clin Orthop Relat Res 1998;(346):95-103

33 Murphy M, Walczak MS, Thomas AG, Silikas N, Berner S, Lindsay R. Toward optimizing dental implant performance: surface characterization of Ti and TiZr implant materials. Dent Mater 2017;33(1):43-53

34 Wennerberg A, Galli S, Albrektsson T. Current knowledge about the hydrophilic and nanostructured SLActive surface. Clin Cosmet Investig Dent 2011;3:59-67

35 Smeets R, Stadlinger B, Schwarz F, et al. Impact of dental implant surface modifications on osseointegration. BioMed Res Int 2016;2016:6285620 